\title{
How heat transfer efficiencies in turbulent thermal convection depend on internal flow modes
}

\author{
KE-QING XIA
}

Department of Physics, The Chinese University of Hong Kong,
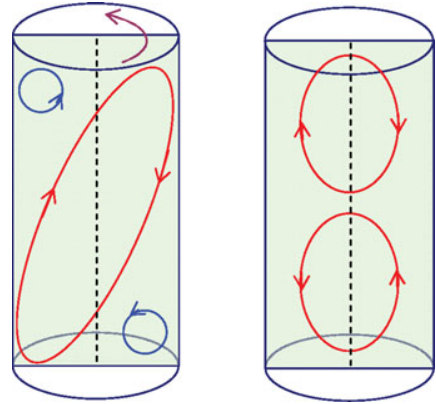

Shatin, Hong Kong, China

How internal flow states can influence the global transport properties in a turbulent system has always been an intriguing question. Weiss \& Ahlers (J. Fluid Mech., this issue, vol. 676, 2011, pp. 5-40) have provided an example by measuring the instantaneous Nusselt number in turbulent Rayleigh-Bénard convection and correlating it to the different modes of large-scale flow.

Key words: heat transport, internal flow states, turbulent convection

\section{Introduction}

The phenomenon of turbulent thermal convection occurs in geophysical and astrophysical systems such as those in the planets and stars, in the atmosphere and oceans and in the mantle and core of the Earth. As an important class of turbulent flows, thermally-driven turbulence differs from inertia-driven turbulence in many ways. Thus, in addition to its relevance to a wide range of natural phenomena, studying convective turbulence can also provide new insights into, and perspectives on, the general turbulence problem itself. As perhaps the simplest system for modelling the convection problem, the Rayleigh-Bénard (RB) system, a fluid layer heated from below and cooled from above, has become a paradigm for thermal convections. A full understanding of convective turbulent flows in this system will thus shed light on a wide range of more complicated convection problems occurring in nature (Ahlers, Grossmann \& Lohse 2009; Lohse \& Xia 2010). In the RB system the dynamics of the flow is determined by the geometry of the cell and two dimensionless parameters: the Rayleigh number $R a=\alpha g \Delta H^{3} /(\nu \kappa)$ and the Prandtl number $\operatorname{Pr}=\nu / \kappa$, where $\Delta$ is the temperature difference across the fluid layer of thickness $H, g$ is the acceleration due to gravity and $\alpha, v$ and $\kappa$ respectively, are the volume expansion coefficient, kinematic viscosity and thermal diffusivity of the fluid.

A prominent feature of the turbulent RB system is the presence of a large-scale circulatory flow (LSC), which is self-organized from thermal plumes that erupt from the top and the bottom thermal boundary layers (Xi, Lam \& Xia 2004). Owing to its many intriguing dynamic features such as coherent oscillations and occasional flow cessations and reversals, the LSC has been studied intensely in recent years. One important reason for the interest in the flow dynamics of the LSC is the possible connection between the internal flow state of the LSC and the efficiency of heat 
transfer. As shown clearly in Weiss \& Ahlers (2011) different internal flow states can indeed produce different heat transfer efficiencies.

\section{Overview}

In $\mathrm{RB}$ convection, the most well-studied geometry is an upright cylinder with an aspect ratio (diameter over height) $\Gamma=1$, in which the dominant large-scale structure is a single circulating roll that spans the height of the convection cell. In contrast, flow dynamics and structures in $\Gamma=0.5$ cells have been studied much less. But turbulent convection in this configuration is no less important. In fact, in the search for the Kraichnan ultimate regime (Kraichnan 1962), many of the heat transport studies utilize the $\Gamma=0.5$ geometry in order to achieve ever higher values of $R a$. From the definition of $R a$ it is easy to see why. For a given fluid and given lateral dimension of the system, which for practical reasons cannot become very large, it is practically more convenient and efficient to achieve larger $R a$ by increasing the height $H$ of the convection cell.

The dominance of the single-roll large-scale flow structure may seem reasonable in $\Gamma=1$ aspect ratio cell, but would it still be the stable and preferred structure in smaller aspect ratio cells, especially when $R a$ becomes very large? Furthermore, the LSC in the $\Gamma=1$ cell has exhibited a wealth of interesting dynamic features such as flow cessations and reversals and torsional and sloshing oscillations. Do they also exist in the $\Gamma=0.5$ cell? Another question is whether there is any connection between the flow structure and the efficiency of heat transfer, and if the answer is yes, which structure is more efficient for heat transfer? These issues were first addressed by Xi \& Xia $(2007,2008 a, b)$ in a series of studies of the flow structures and dynamics, which have revealed that some of the dynamic properties observed in $\Gamma=1$ cell are also present in the $\Gamma=0.5$ geometry. They also discovered the existence of a double-roll mode (DRM), i.e. one roll stacked on top of the other (shown on the right in the figure by the title) and identified several possible routes of transition between the DRM and the single-roll mode (SRM; shown on the left in the figure by the title). These flow mode transitions have now been confirmed by Weiss and Ahlers, but over a much wider range of $R a$. They discovered that the SRM becomes the dominant mode with increasing $R a$ while the contribution from the DRM diminishes, and also that below a certain $R a\left(\sim 7 \times 10^{8}\right)$, the DRM becomes more prevalent than the SRM. This might be caused by the fact that with the weakening of the buoyancy force that comes with reduced $R a$, the thermal plumes that form and drive the LSC do not have sufficient energy to reach the opposite plate of the cell to form a single-roll LSC.

Weiss and Ahlers also discovered that when the LSC is in the SRM, its top and bottom parts oscillate horizontally (i.e. out of the vertical circulation plane, denoted by the dark red arrow in the figure by the title) with a phase difference of $\pi$. This so-called torsional mode was first observed by Funfschilling \& Ahlers (2004) in $\Gamma=1$ and is one of the more striking examples demonstrating the rich dynamics in the deceptively simple RB system. It was thought to be absent in the $\Gamma=0.5$ cell from the studies of $\mathrm{Xi} \& \mathrm{Xia}(2008 a)$. A possible reason for their missing this mode initially is that data in $\Gamma=0.5$ case is more noisy than $\Gamma=1$ case, owing to the fact that the LSC is not always in the more stable SRM. Thus, it is no small feat that Weiss and Ahlers were able to extract the torsional mode from the noisy data.

A long-standing question in RB turbulence is whether there exists a connection between the flow state of the LSC and the Nusselt number. More generally, the question is: do global transport properties depend on internal flow states in 
(a)

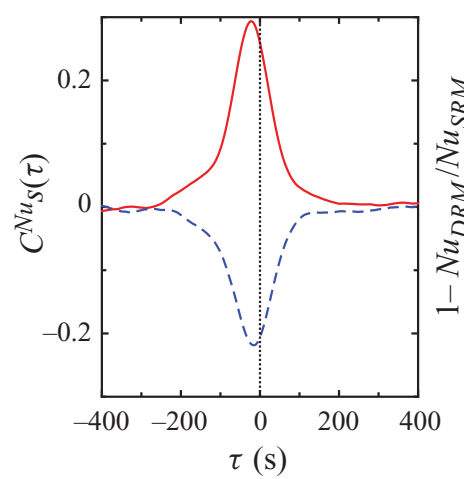

(b)

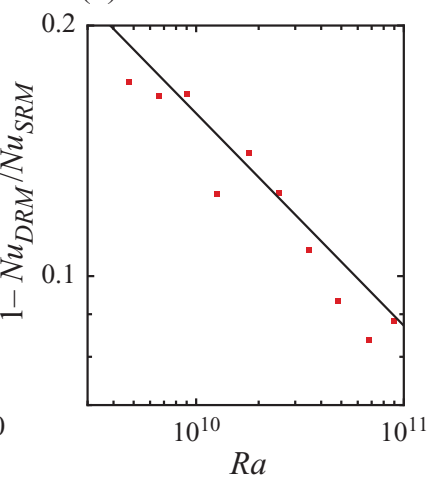

(c)

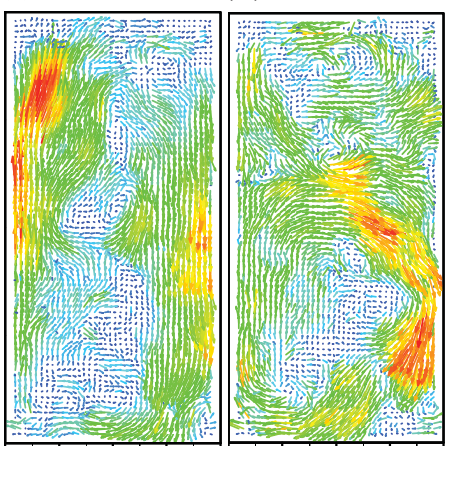

FiguRE 1. (a) Cross-correlation functions between $N u(t)$ and a state function $S(t)$ for the SRM (solid line) and the DRM (dashed line), where $S(t)$ is 1 when LSC is in the corresponding state and zero otherwise. (b) Normalised difference between $N u$ in the SRM and $N u$ in the DRM on logarithmic scales (figure from Weiss \& Ahlers 2011). (c) and (d) PIV-measured instantaneous velocity maps giving direct visualizations of the SRM and the DRM respectively (figure from Xi \& Xia 2008b).

turbulence? This issue was first raised by the finding of Roche et al. (2002) in their experiment conducted in low temperature helium gas in a cylindrical cell of $\Gamma=1 / 2$, which showed that the measured $N u$ exhibited bimodal behaviour, i.e. the repeatedly measured $N u$ for the same value of $R a$ fell onto two bands separated by a few per cent, and it was suggested that this could be caused by the large-scale flow switching between the one-roll and two-roll structures.

Using an artificially generated flow mode in a $\Gamma=1 / 2$ cylindrical cell, Sun, Xi \& Xia (2005) were able to produce a bimodal- $N u$ behaviour and showed that a particular value of $N u$ can be unambiguously associated with a specific large-scale flow mode in the convection cell. But this is not direct evidence to link the SRM and DRM with different $N u$. Later, Xi \& Xia (2008a) determined $N u$ conditioned on a particular flow and found that $N u_{S R M}$ is larger than $N u_{D R M}$. But the difference is only about $0.2 \%$ and so it may not be viewed as a proof beyond doubt. By determining the Nusselt number associated with SRM and with DRM separately, Weiss and Ahlers show that $N u_{S R M}$ is indeed larger than $N u_{D R M}$. As can be seen from figure 1(a), the measured $N u(t)$ is correlated positively with the SRM and negatively with the DRM, implying that $N u$ is higher than the average when the LSC is in SRM and lower than the average when it is in DRM. To give direct visualizations of the SRM and DRM, instantaneous velocity maps measured by particle image velocimetry (PIV) are also shown in the figure. From figure $1(b)$ one can see that the difference between $N u_{S R M}$ and $N u_{D R M}$ depends on $R a$, which varies from $\sim 1.6 \%$ to $\sim 0.9 \%$ from $R a \simeq 10^{10}$ to $R a \simeq 10^{11}$. This difference is larger than the experimental uncertainties and thus provides the clearest and most convincing evidence so far that the SRM is indeed more efficient for turbulent heat transport than the DRM.

\section{Future}

With the connection between the internal flow states and the global heat transport now firmly established, future works should be focused on how to use this finding 
to bring about a quantitative understanding on the bimodality or multimodality of the measured $\mathrm{Nu}$ under nominally identical conditions. Unfortunately, the present finding cannot be applied straightforwardly to the existing data. For example, the bimodal values of $N u$ found by Roche et al. (2002) differ by about $7 \%$ which appears to be insensitive to $R a$ over the range $2 \times 10^{7}-2 \times 10^{10}$. While this appears to be incompatible with the findings of Weiss and Ahlers, we note that the situation is more complicated. In Roche et al.'s experiment, the value of $\mathrm{Pr}$ is not constant but varies over a wide range (from $\sim 0.7$ to $\sim 10$ ), whereas the different $N u_{S R M}$ and $N u_{D R M}$ behaviour found by Weiss and Ahlers was observed between $R a \simeq 10^{10}$ and $\simeq 10^{11}$ and at a fixed $P r=4$.3. Furthermore, their analysis of data measured at $P r=0.7$ indicates that the DRM is unlikely to be important for low Pr. Therefore, a more systematic study on the $P r$-dependency of the SRM and DRM is required before a full understanding of the experimentally observed bimodality in $N u$ can be achieved. Another issue that deserves further investigation is to understand why the difference between $N u_{S R M}$ and $N u_{D R M}$ found by Xi \& Xia (2008b) is about five times smaller than that found by Weiss and Ahlers at comparable values of $R a$ and $P r$. There have been speculations that the single-roll LSC will breakdown beyond a certain but unspecified value of $R a$ - the heat transport in the RB system will presumably then enter a different regime. Again, this issue has to be addressed with the $P r$-dependency taken into consideration.

\section{References}

Ahlers, G., Grossmann, S. \& Lohse, D. 2009 Heat transfer and large scale dynamics in turbulent Rayleigh-Bénard convection. Rev. Mod. Phys. 81, 503-537.

Funfschilling, D. \& Ahlers, G. 2004 Plume motion and large scale circulation in a cylindrical Rayleigh-Bénard cell. Phys. Rev. Lett. 92, 194502.

Kraichnan, R. H. 1962 Turbulent thermal convection at arbitrary Prandtl number. Phys. Fluids 5, $1374-89$.

LoHSE, D. \& XIA, K.-Q. 2010 Small-scale properties of turbulent Rayleigh-Bénard convection. Аnпи. Rev. Fluid Mech. 42, 335-64.

Roche, P. E., Castaing, B., Chabaud, B. \& Hebral, B. 2002 Prandtl and Rayleigh numbers dependences in Rayleigh-Bénard convection. Europhys. Lett. 58, 693-698.

SuN, C., XI, H.-D. \& XIA, K.-Q. 2005 Azimuthal symmetry, flow dynamics, and heat flux in turbulent thermal convection in a cylinder with aspect ratio one-half. Phys. Rev. Lett. 95, 074502.

WeIss, S. \& Ahlers, G. 2011 Turbulent Rayleigh-Bénard convection in a cylindrical container with aspect ratio $\Gamma=0.50$ and Prandtl number $P r=4.38$. J. Fluid Mech. 676, 5-40.

XI, H.-D., LAM, S. \& XIA, K.-Q. 2004 From laminar plumes to organized flows: the onset of large-scale circulation in turbulent thermal convection. J. Fluid Mech. 503, 47-56.

XI, H.-D. \& XIA, K.-Q. 2007 Cessations and reversals of the large-scale circulation in turbulent thermal convection. Phys. Rev. E 76, 036301.

XI, H.-D. \& XIA, K.-Q. 2008 a Azimuthal motion, reorientation, cessation and reversal of the largescale circulation in turbulent thermal convection: a comparison between aspect ratio one and one-half geometries. Phys. Rev. E 78, 036326.

XI, H.-D. \& XIA, K.-Q. $2008 b$ Flow mode transitions in turbulent thermal convection. Phys. Fluids 20, 055104. 